\title{
Application of Remote Sensing Image in Civil Engineering
}

\author{
Ting Wang ${ }^{1 *}$, Lizhu $\mathrm{Ma}^{1}$, and Qinghui $\mathrm{Qi}^{1}$ \\ ${ }^{1}$ Civil Engineering, Shenyang Urban Construction University, Shenyang, Liaoning, 110167, CHINA
}

\begin{abstract}
Civil engineering occupies an unwavering position in the scientific and technological field. It not only has a deep tradition, but also has unlimited development potential in the future, and it covers numerous academic and application fields. With the advancement of science and technology, remote sensing technology is more and more widely used in people's daily production and life. In civil engineering construction and research, remote sensing imagery also plays an important role as the basic data. Therefore, remote sensing technology has shown important support in land utilization, land construction planning, and coverage monitoring. According to the actual needs of civil engineering, this paper extracts targeted land information from satellite remote sensing images in a certain area, and analyzes the practical and reasonable needs of civil engineering construction.
\end{abstract}

\section{Research background}

Land resources are the basis for human existence and development, and they are the basic land for human survival and management of social and economic activities. The true and accurate reflection of land use and coverage data is closely related to the development of land and resources management, at the same time, it is closely related to the country's macro-control, and it is the fundamental and important basis for control. With the population growth and economic and social development, the pressure on the use of land resources is increasing, and the contradictions between people and land are becoming increasingly prominent. In the regional "population-resource-environmentdevelopment" (PRED) composite system, land resources are at the core of any other resources that cannot be replaced. At the same time, land resources are immovable and non-renewable resources. The effective use and rational planning of land resources are the key elements of economic development. ${ }^{[1]}$

Remote sensing is a technology that uses non-contact methods and uses hyperspectral technology to observe ground objects to obtain its information. Since the early days of development, based on the multidisciplinary foundation of time, space, computer, physics and geoscience, it has rapidly developed into a new scientific and comprehensive technology. Remote sensing data has many advantages, such as high real-time, wide coverage, rich and objective information, so it has been widely used in many fields such as land use, land planning and construction, resource exploration, environmental monitoring, and atmospheric oceans. It is playing an increasingly important role in social and economic development.

Civil engineering disciplines cover many aspects such as soil mechanics, basic engineering, highway / railway engineering, tunnel engineering, fluid mechanics, open channel / underdrain engineering, bridge building, dam building, and building construction. The remote sensing technology of surveying engineering discipline can show the foundation support function in the civil engineering construction process.

In civil engineering construction, when selecting routes in road engineering or pipeline engineering, the basic situation of the construction area is an indispensable reference file. In order to plan the project and prepare a budget, it is necessary to design a construction plan and draw up a project budget based on factors such as on-site facilities, topography, and other factors. Land use information can reflect the basic situation of the construction area. With accurate ground use information, an appropriate computer-aided design system can be applied to plan and compare the feasibility and investment ratio of multiple possible routes. Several routes with less engineering cost and lower cost are selected in advance. It can further cooperate with factors such as economic benefits and defense measures to choose the best route.

\section{Research methods}

\subsection{Extraction of land use information}

\footnotetext{
* Corresponding author: lngd1014704@163.com
} 


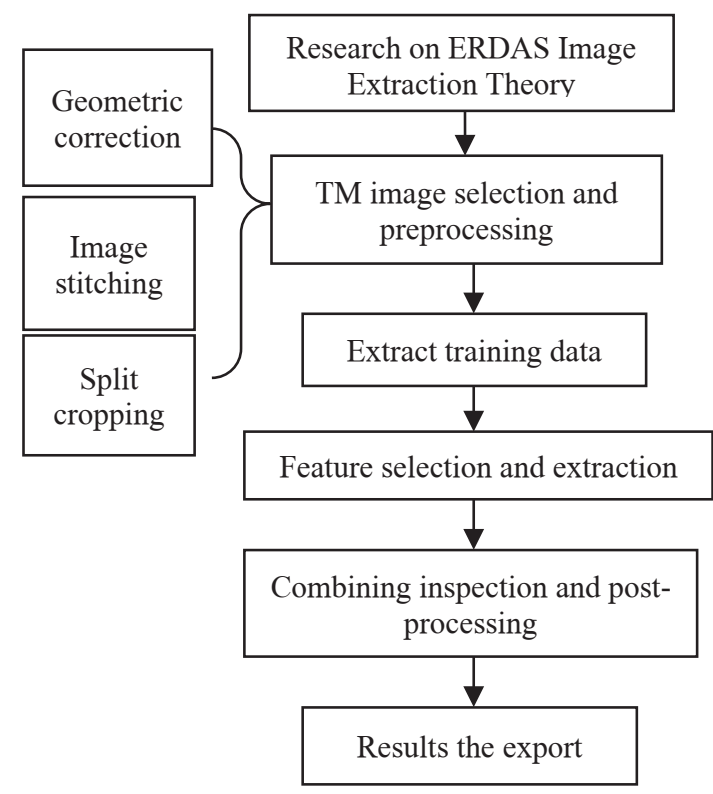

Fig. 1. Technical route.

\subsubsection{Data source}

USGS is used to find image data with ideal conditions, and Landsat TM remote sensing image is used for preprocessing and classification to obtain land use information. TM data consists of 7 bands with a resolution of 30 meters. The image selection criteria is better reflection of ground objects, distinct land use effects, and higher image resolution. Ancillary data sources are vector maps of administrative boundaries in the area and so on.

\subsubsection{Interpretation of remote sensing images}

Erdas software was used to preprocess the data in the engineering area, including geometric correction, band combination, influence stitching and cropping.

(1) Geometric correction

The geometric errors in remote sensing images are mainly reflected in the image displacement, rotation, and distortion and offset of the pixel ground relative to the actual location. In order to ensure the accuracy of remote sensing image processing and have standard geospatial coordinates when performing spatial analysis, the study of geometric correction of remote sensing images to reduce errors is mainly divided into three steps: one is to collect appropriate ground control points; the other is to perform spatial transformation of the image according to the ground control points; the third is to interpolate and resample the grayscale values of the spatially transformed image. ${ }^{[2]}$

(2) Band combination

Different bands in TM images contain different amounts of information, and the general measurement standard is determined based on the range of brightness values. In general, the information content in the midinfrared band is the most abundant, and the amount of information in the visible light band is less than that in the infrared band. Among the seven bands of TM images, the correlation between the three visible light bands TM1, TM2, TM3 and the two mid-infrared bands TM5 and TM7 is very high, that is, the repetition is high; the correlation between TM4 and other bands is very low.

According to the characteristics of each band of the image and the purpose of the study, the current status of land use is classified. In this study, the combination of the bands TM3, TM4, and TM5 is relatively ideal and easy to visually interpret.

(3) Image stitching

The remote sensing image obtained by the sensor is segmented. According to the actual situation of the project area, it is just in the adjacent multiple images. By stitching the images, a complete research area is obtained.

(4) Image cropping

Because the obtained image is beyond the scope of the study area, in order to reduce the data storage space and reduce the extra processing data time, the image is first cropped and then the image is classified and extracted.

ERDAS is used to crop the image. In this step, two processing methods are used: regular frame cropping and irregular frame cropping. The former uses the coordinates of the two endpoints of the diagonal segment to delimit the trimming range; the latter method uses the establishment of a polygon area of interest (AOI) to delimit the required study area.

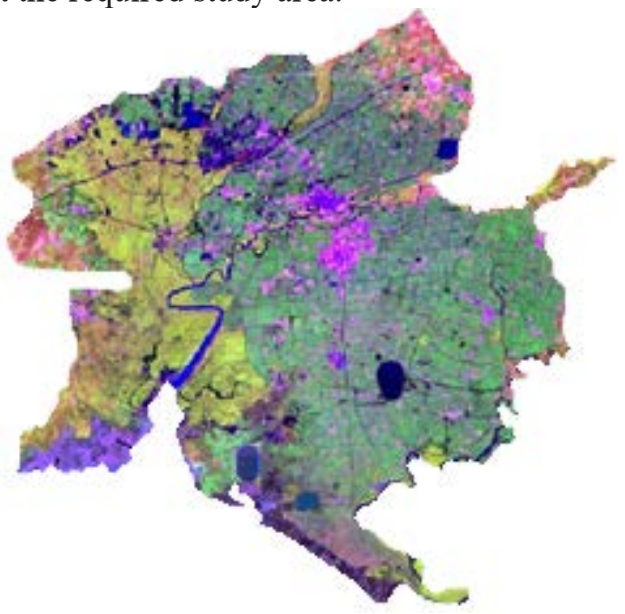

Fig. 2. Image cropping results.

\subsubsection{Information data extraction}

Under the same conditions (lighting, terrain, etc.), different features will show differences in spectral information and spatial information characteristics. The process of dividing all pixels in an image into multiple categories according to the presented differences is defined as image classification. According to the prior type and training samples provided before classification, it can be divided into two methods: supervised classification and unsupervised classification. Unsupervised classification does not need to have a wealth of prior knowledge. Based on the unique spectral characteristics of the pixels, it uses an iterative selforganizing data analysis (ISODATA) algorithm to classify certain pixels into different categories based on their similar characteristics; The premise is to have 
certain prior knowledge of the information attributes in the image, select and formulate samples of the features to be classified from the image, establish a classification template, and realize automatic recognition on this basis. ${ }^{[3]}$

According to the relevant provisions of the national land use classification standard and the specific conditions and characteristics of the test area, the ground features in this area are divided into seven types: paddy fields, dry land, residential land, water bodies, grassland, forest land, and garden land. The method used in this study to classify remote sensing images is supervised classification. After defining the classification template and performing supervised classification, the land use map of the region is obtained, as shown in Figure 3.

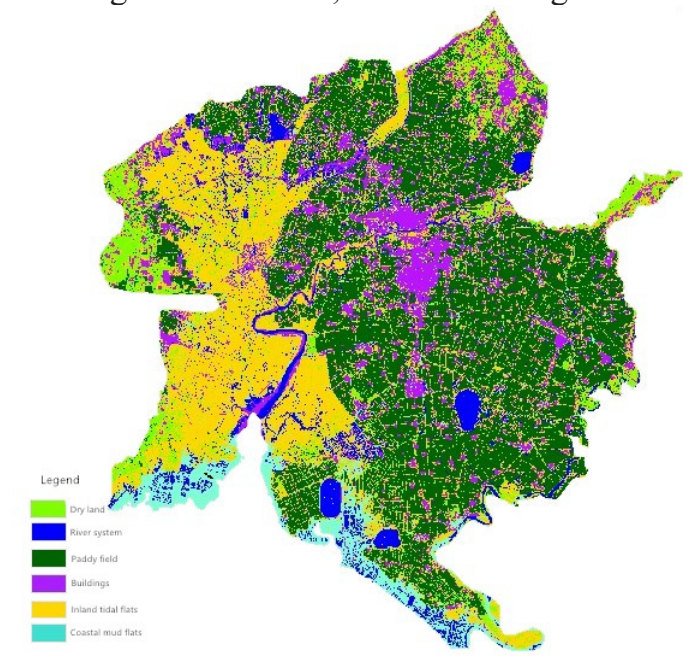

Fig. 3. Image classification results map.

\subsubsection{Classification accuracy}

Remote sensing image classification accuracy test is an indispensable link in remote sensing classification technology. Performing the accuracy test can determine the validity of the classification model thereby transform the classifier and improve the classification accuracy; on the other hand, the information in the classification result can be obtained correctly and effectively according to the accuracy of the classification results. The classification accuracy of remote sensing image is usually evaluated by comparing the classification map with standard data, maps or ground measured values, and expressing the classification accuracy as a percentage of correct classification. The evaluation of position accuracy is to check the classification accuracy while considering the classification category and its spatial position. The method used in this study is the confusion matrix method, which uses Kappa coefficients to evaluate the accuracy of the entire classification map, and conditional Kappa coefficients to evaluate the accuracy of a single category, and $t$ is also the main accuracy evaluation method for remote sensing classification. If the classification image is very different from the original image, its Kappa coefficient is small.

According to the classification accuracy evaluation, the image classification in this area is Overall Classification Accuracy $=85.00 \%$, Overall Kappa
Statistics $=0.7719$.

Table 1. The relation of Kappa coefficient to classification accuracy.

\begin{tabular}{|c|c|}
\hline Kappa coefficient & Classification quality \\
\hline$<0.00$ & Bad \\
\hline $0.00-0.20$ & Poor \\
\hline $0.20-0.40$ & Ordinary \\
\hline $0.40-0.60$ & Good \\
\hline $0.60-0.80$ & Perfect \\
\hline $0.80-1.00$ & Excellent \\
\hline
\end{tabular}

\subsubsection{Statistics on land use status}

Statistics on the status of land use types are used as the basis for civil engineering construction, and Landsat TM in this area is the main source of information. The results of the current status of land use in this area are obtained through remote sensing interpretation. Then, through the form summary function of ArcGIS, Statistics of current land use types are obtained.

The statistical data of the current status of land use in this area is a quantitative representation of various types of land use. The statistical data is based on the remote sensing interpretation results of the current status of the land use in this area and is summarized in the form of the GRID-CODE field in ArcGIS. Table 2 shows the statistical results of the current land use data in this area.

Table 2. Data statistical results of present land use.

\begin{tabular}{|c|c|c|}
\hline \multicolumn{2}{|c|}{ Land class } & $\begin{array}{c}\text { statistical ma } \\
\text { gnitude }\end{array}$ \\
\hline \multirow{2}{*}{ Plowland } & paddy field & \multirow{2}{*}{$111099 \mathrm{hm}^{2}$} \\
\cline { 2 - 2 } $\begin{array}{c}\text { Construction } \\
\text { land }\end{array}$ & $\begin{array}{c}\text { Land for residence, } \\
\text { commerce, etc. }\end{array}$ & $535.00 \mathrm{~km}^{2}$ \\
\hline \multirow{3}{*}{ Water area } & River system & \multirow{2}{*}{$765.87 \mathrm{~km}^{2}$} \\
\cline { 2 - 2 } & Coastal mud flats \\
\cline { 2 - 2 } & Inland tidal flats & \\
\hline
\end{tabular}

\subsection{Application of civil engineering projects}

Land use results from targeted remote sensing image interpretation can meet the needs of different engineering construction projects.

Remote sensing technology provides a new method for engineering surveys. Its macroscopicity, intuitiveness, and comprehensiveness cannot be achieved by any other ground survey. Its functions can be summarized as follows:

(1) Make up for the lack of regional land information. Utilizing the land type data in the area to update the problems about aging data, lack of geomorphological content of the survey area, and incomplete data, the implementation of remote sensing surveys, especially remote sensing surveys in the preliminary work, and it can make up for the shortcomings in this area.

(2) Effectively identify major geomorphological 
issues and stabilize engineering solutions. Combined with the actual economic and human development of the area, reasonable planning of the development route, scale, location, etc. of the corresponding construction projects. Through remote sensing image information extraction, it can provide basic information for project site selection, and a more comprehensive land use can be obtained in a larger area Information, you can find the major engineering geomorphology and land types of the survey area from a macro perspective, as well as the major engineering planning problems of major projects (such as long bridges, tunnels, interchanges, etc.), and provide a more reliable basis and comparison for program selection Choose opinions.

(3) Due to the irreversibility of project construction and its irreversible impact on the environment, a thorough understanding of environmental factors, attention to the surrounding conditions of the project, and respect for its laws are the most fundamental principles of site selection.

1) The principle of avoidance

Wherever the agency operates, it will objectively generate economic input, in order to avoid disputes, ensure stability, maximize benefits in economic development, agency needs to combine local economic development according to actual land use types, pay attention to people 's livelihood, and where conditions allow, avoiding sensitive areas is the most economical and safest method.

\section{2) Feasibility principle}

For example, highways are susceptible to terrain conditions. Therefore, when selecting a route, we should first find out the basic factors such as the type, shape, scale, and potential impact of the land that the route crosses. Try to choose favorable terrain to ensure that the scheme is feasible under the existing technical and economic conditions. The feasibility principle is the basic guarantee for the successful completion of the entire construction project.

3) Security Principles

With the humanistic design concept deeply rooted in the hearts of the people, departments at all levels have clearly required that project construction must reflect the "people-oriented" humanistic spirit. Ensuring the safety of people's lives and property at all stages of the project is one of the factors that must be considered in engineering design. The safety mentioned here has two meanings: one is the safety during the construction stage, and the other is the safety during the later operation stage.

4) Economic principles

Regional construction investment is huge. At the current stage of the development level of the national economy, cost reduction and investment savings are the basic guarantee to ensure the smooth implementation of the project, and an important factor to be paid attention to in the process of construction. Therefore, for the purpose of achieving operational benefit maximization, saving investment and low maintenance costs, we must implement the economical geological site selection.

5) Principles of sustainable development

Many factors should be considered in large-scale civil engineering projects. In the overall design, consideration should be given to the first-time design, supporting measures to facilitate the connection with the second-phase project, and advocating the integration of the overall planning, which considers the implementation of the first phase, and reserves space for the secondphase project to ensure the effective implementation of the first- and second-phase projects and the overall function.

\section{Conclusion}

In the early stages of civil engineering and the stage of project development, regional data are emphasized. In the process of collecting topographical and geomorphological data, if traditional methods are used, it is not only inefficient, but also requires a lot of manpower and financial resources. The long measurement time can easily cause extension of project construction period. Through the application of remote sensing technology, various raw data can be accurately, quickly and efficiently collected. While ensuring the quality and efficiency of engineering surveys, it can also provide scientific information and data for engineering construction, laying a good foundation for the smooth development of engineering construction work. ${ }^{[4]}$

When remote sensing technology is applied to detect the geometry and radiation characteristics of surface objects, the characteristics related to civil engineering applications are:

(1) Do not touch the target. For areas not in direct contact, telemetry is the only method remaining. Another example is that in the material load deformation test, it is not suitable to make additional contact with the test object and introduce unknown external forces, or because of security considerations, close-up photography can accurately measure the amount of detailed deformation beyond a few meters.

(2) Obtain a complete two-dimensional image record in a limited period of time. When necessary, it is easy to re-test or even re-test on the image.

(3) Digital telemetry images to facilitate computer data processing. The three-dimensional overlay image can be used to derive the three-dimensional digital model. The number of to-be-fixed points can be increased arbitrarily, so that the model can approach the real object without limit.

(4) If you want to improve the accuracy of telemetry, you can shorten the distance between the camera and the object. But because the image scale becomes larger, the area covered by the image is relatively reduced.

(5) According to multi-band remote sensing information, the classification of features can be automated. Remote sensing technology is obtained from images, and then processed to obtain valuable information. The extracted information can be used to its maximum value through image interpretation and the effective use and interpretation of relevant professionals. As an auxiliary technology in the field of civil engineering, remote sensing technology is applied from a professional perspective and combined in multiple fields to achieve common development. 


\section{References}

1. L. Wang, J. L. Lin, S.L. Wang, Y.B. Lin, Environmental Technology , 04(2018)

2. Y.M. Tan, W. Xia, Geomatics and Spatial Information Technology, 04(2014)

3. H.W. Bai, M.Y. Wu, Y. Ge, Journal of Shenyang University (Natural Science), 01(2016)

4. Z.F. Zhu, W. Zhang, Automation Technology and Application, 12(2016)

5. J. Shi, X.W. Deng, Sichuan Cement, (03)2019

6. H.Q. Qiang, Y. Zhao, Research on Urban Construction Theory (Electronic Version), (8)2017 